\title{
Nonlinear Oscillations of a Mass Attached to Linear and Nonlinear Springs in Series Using Approximate Solutions
}

\author{
Beyza Bostanc1 ${ }^{1}$, M. M. Fatih Karahan²* \\ ${ }^{1}$ Department of Mechanical Engineering, Natural and Applied Science, Manisa Celal Bayar University, Campus of Şehit \\ Prof. Dr. İlhan Varank, 45140, Manisa,Turkey \\ ${ }^{2}$ Department of Mechanical Engineering, Faculty of Engineering, Manisa Celal Bayar University, Campus of Şehit Prof. \\ Dr. İlhan Varank, 45140, Manisa,Turkey \\ *fatih.karahan@cbu.edu.tr \\ Received: 23 February 2018 \\ Accepted: 13 June 2018 \\ DOI: $10.18466 /$ cbayarfbe. 397802
}

\begin{abstract}
Nonlinear oscillations of a mass with serial linear and nonlinear stiffness on a frictionless surface is considered. Equation of motion of the considered system is obtained. For analysing of the system, relatively new perturbation method that is named Multiple Scales Lindstedt Poincare (MSLP) and classical multiple scales (MS) methods are used. Both approximate solutions are compared with the numerical solutions for weakly and strongly nonlinear systems. For weakly nonlinear systems, both approximate solutions are in excellent agreement with numerical simulations. However, for strong nonlinearities, MS method is not give reliable results while MSLP method can provide acceptable solutions with numerical solutions.

Keywords: Multiple Scales Lindstedt Poincare (MSLP) method, Nonlinear Oscillation, Nonlinear Stiffness, Perturbation Methods.
\end{abstract}

\section{Introduction}

To find exact analytical solution of the physical systems is usually impossible for most of the time. For this reason, approximate analytical methods are developed for solving mathematical models corresponding to physical problems. Perturbation methods are used efficiently for finding approximate analytical solutions in many physical problems $[1,2,3]$. Perturbation methods are valid for weakly nonlinear systems due to the assumption of small parameters. To overcome this deficiency, methods, such as linearized perturbation method [4], the Lindstedt-Poincare method with modified frequency expansion [5], the parameter expanding method [6] and homotopy perturbation [7] were developed within time.

A relatively new method which gives valid solutions for both weak and strong nonlinear systems [8-13] has been developed. The method is called Multiple Scales Lindstedt Poincare (MSLP) which is combination of multiple scales and Lindstedt Poincare tecniques.

In this work, MSLP method is applied to system of a mass with serial linear and nonlinear stiffness for the first time. Approximate analytical solutions are obtained via MS and MSLP methods. The obtained solutions are compared with numerical integration solutions. It has been found that the
MSLP method provides acceptable solutions for strong nonlinearities, while MS solutions are not suitable for the strong nonlinearities.

Finally, for the case of free vibration of a system of a mass with serial linear and nonlinear stiffness, some works are mentioned [14-17]. Telli and Kopmaz [14] applied Lindstedt Poincare and harmonic balance methods to above mentioned system. Both solutions are compared with numerical results. It is found that numerical and obtained analytical solutions are in very good agreement for weak nonlinearities. Linearized harmonic balance method is applied to the governing equation of motion by Lai and Lim [15]. Although the method has the ability to generate highly accurate frequencies, applications are limited to conservative systems. Conversely, MSLP is employed in a broader range of vibration problems with simpler calculations. Hoseini et al. [16] and Bayat et al. [17] employed homotopy analysis and He's variational approach methods to the same problem respectively. In their method, frequencies, although with acceptable accuracy, are not given in closed functional forms. 


\section{Material and Methods}

2.1. A Nonlinear Oscillation of a Mass Attached to Linear and Nonlinear Springs in Series

In this section, free vibration of a system of mass with serial linear and nonlinear stiffness is examined. First, the equation of motion is produced for the mentioned nonlinear system. Multiple Scales (MS) and Multiple Scale Lindstedt Poincare (MSLP) are applied to solve the nonlinear system.

\subsection{Equation of Motion}

In Figure 1, a system that includes a mass attached to linear and nonlinear springs in series is shown.

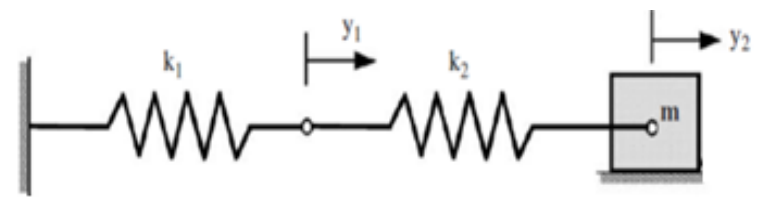

Figure 1. Nonlinear free vibration of a system of mass with serial linear and nonlinear stiffness on a frictionless contact surface.

The stiffness coefficients of the springs are $k_{1}$ and $k_{2}$ respectively. The nonlinear spring includes cubic nonlinearities. The relationship between the force and the deflection of the nonlinear spring is as follows.

$$
F_{2}=k_{2} x=k_{3} x+\lambda x^{3}=k_{3} x+\varepsilon k_{3} x^{3}
$$

where

$$
\varepsilon=\frac{\lambda}{k_{3}}
$$

in which $\lambda$ is coefficient of nonlinear portion of second spring force and $k_{3}$ is coefficient of linear portion of this nonlinear spring force. The cubic nonlinear characteristic determines that hardening/softening behaviour. If $\varepsilon>0$, the spring shows a hardening behaviour. Likewise, it is indicated that softening spring for the case $\varepsilon<0$. Parameter $\varepsilon$ is employed as a perturbation in this system.

The displacements between the connection point of the spring and mass are defined by $y_{1}$ and $y_{2}$. In Figure 1 , the displacements of the springs are expressed by $y_{1}$ and $\left(y_{2}-y_{1}\right)$ respectively. In this case, the displacement of mass $m$ will be $y_{1}+\left(y_{2}-y_{1}\right)=y_{2}$. The equation of motion of the single-degree-of-freedom system will be found with Lagrangian $(L)$. First, the system's potential and kinetic energy will be written as follows respectively.

$$
\begin{aligned}
& V=\frac{1}{2} k_{1} y_{1}^{2}+\frac{1}{2} k_{2}\left(y_{2}-y_{1}\right)^{2} \\
& T=\frac{1}{2} m \dot{y}_{2}^{2}
\end{aligned}
$$

Lagrange's equation is written to obtain the equation of motion of the system.

$$
\frac{d}{d t}\left(\frac{\partial L}{\partial \dot{q}_{i}}\right)-\frac{\partial L}{\partial q_{i}}=Q_{i}, i=1,2
$$

where $q_{i}$ and $Q_{i}$ express respectively general coordinates of system and the sum of the forces acting on these coordinates. Because it is a conservative system which will be solved, Equation (5) must be equal to zero [18].

$$
\frac{d}{d t}\left(\frac{\partial L}{\partial \dot{y}_{i}}\right)-\frac{\partial L}{\partial y_{i}}=0
$$

The quantity $T-V$ is called the Lagrangian $L$.

$$
L=T-V
$$

Substituting into Equation (6) and the following equations are obtained.

$$
\begin{aligned}
& k_{1} y_{1}-k_{2}\left(y_{2}-y_{1}\right)=0 \\
& m \ddot{y}_{2}+k_{2}\left(y_{2}-y_{1}\right)=0
\end{aligned}
$$

From Equation(8), the relation between $y_{1}$ with $y_{2}$ are obtained as follows.

$$
y_{1}=\frac{k_{2}}{k_{1}+k_{2}} y_{2}
$$

Equation (10) is written instead in Equation(9).

$m \ddot{y}_{2}+\frac{k_{1} k_{2}}{k_{1}+k_{2}} y_{2}=0$

where the dots denote the derivation with respect to time. Equation of motion is obtained as follows

$m \ddot{y}_{2}+k_{e q} y_{2}=0$

where $k_{e q}=\left(\frac{k_{1} k_{2}}{k_{1}+k_{2}}\right)$ is equivalent stiffness of the springs. The nonlinear spring force in Equation (1) is substituted into Equations (8) and (9) and equation of motion is obtained as follows

$$
\begin{aligned}
& k_{1} y_{1}-k_{3}\left(y_{2}-y_{1}\right)-\varepsilon k_{3}\left(y_{2}-y_{1}\right)^{3}=0 \\
& m \ddot{y}_{2}+k_{3}\left(y_{2}-y_{1}\right)+\varepsilon k_{3}\left(y_{2}-y_{1}\right)^{3}=0
\end{aligned}
$$

The new variables $v$ and $u$ are defined as follows.

$y_{1}=v$,

$y_{2}-y_{1}=u$

Inserting the new variables into Equations (13) and(14) one has 


$$
\begin{aligned}
& k_{1} v-k_{3} u-\varepsilon k_{3} u^{3}=0 \\
& m(\ddot{u}+\ddot{v})+k_{3} u+\varepsilon k_{3} u^{3}=0
\end{aligned}
$$

Solving Eq. (16) for $v$ yields

$$
v=\xi u+\xi \varepsilon u^{3}
$$

where

$$
\xi=\frac{k_{3}}{k_{1}}
$$

Equation (18) is derivatived twice with respect to time and substituted into Equation(17). One finds

$$
m \ddot{u}\left(1+\xi+3 \varepsilon \xi u^{2}\right)+6 \varepsilon \xi m u \dot{u}^{2}+k_{3} u+\varepsilon k_{3} u^{3}=0
$$

After algebraic manipulations, Equation (20) yields

$\ddot{u}\left(1+3 \varepsilon \alpha u^{2}\right)+6 \varepsilon \alpha u \dot{u}^{2}+\omega_{0}{ }^{2} u+\varepsilon \omega_{0}{ }^{2} u^{3}=0$

where

$$
\alpha=\frac{\xi}{\xi+1}, \omega_{0}^{2}=\frac{k_{3}}{m(1+\xi)}
$$

and $\omega_{0}$ is natural frequency of the system.

\subsection{Multiple Scales (MS) method}

Slow and fast time scales are

$T_{0}=t, \quad T_{1}=\varepsilon t, T_{2}=\varepsilon^{2} t$

Using

$\frac{d}{d \tau}=D_{0}+\varepsilon D_{1}+\varepsilon^{2} D_{2}+\ldots$,

$\frac{d^{2}}{d \tau^{2}}=D_{0}^{2}+2 \varepsilon D_{0} D_{1}+\varepsilon^{2}\left(D_{1}^{2}+2 \varepsilon D_{0} D_{2}\right)+\ldots$

Substituting the expansions

$$
u=u_{0}\left(T_{0}, T_{1}, T_{2}\right)+\varepsilon u_{1}\left(T_{0}, T_{1}, T_{2}\right)+\varepsilon^{2} u_{2}\left(T_{0}, T_{1}, T_{2}\right)+\ldots
$$

The equations are separated for each order

$u_{0}(0)=a_{0}, \quad D_{0} u_{0}(0)=0$

$O(\varepsilon): D_{0}^{2} u_{1}+\omega_{0}^{2} u_{1}=-2 D_{0} D_{1} u_{0}-3 \alpha u_{0}^{2} D_{0}^{2} u_{0}$

$-6 \alpha u_{0}\left(D_{0} u_{0}\right)^{2}-\omega_{0}{ }^{2} u_{0}^{3}$

$u_{1}(0)=0, \quad\left(D_{0} u_{1}+D_{1} u_{0}\right)(0)=0$

$O\left(\varepsilon^{2}\right): D_{0}{ }^{2} u_{2}+\omega_{0}{ }^{2} u_{2}=-2 D_{0} D_{1} u_{1}-\left(D_{1}^{2}+2 D_{0} D_{2}\right) u_{0}$

$-3 \alpha\left[2 u_{0} u_{1} D_{0}^{2} u_{0}+u_{0}^{2}\left(D_{0}{ }^{2} u_{1}+2 D_{0} D_{1} u_{0}\right)\right]$

$-6 \alpha\left[u_{1}\left(D_{0} u_{0}\right)^{2}+2 u_{0} D_{0} u_{0}\left(D_{0} u_{1}+D_{1} u_{0}\right)\right]-3 \omega_{0}^{2} u_{0}{ }^{2} u_{1}$

The solution of first order is

$u_{0}=A\left(T_{1}, T_{2}\right) e^{i \omega_{0} T_{0}}+c c$

where

$A=\frac{1}{2} a e^{i \beta}$
In terms of real amplitude and phase, the first order solution is

$u_{0}=a\left(T_{1}, T_{2}\right) \cos \left(\omega_{0} T_{0}+\beta\left(T_{1}, T_{2}\right)\right)$

Initial conditions are applied for first order, one has $a(0)=a_{0}, \beta(0)=0$

The secularities are eliminated in the right side of Equation (27)

$-2 i \omega_{0} D_{1} A+\omega_{0}^{2}(3 \alpha-3) A^{2} \bar{A}=0$

Substituting the polar form, real and imaginary parts are separated

$a=a\left(T_{2}\right)$

$\beta=-\frac{(3 \alpha-3)}{8} \omega_{0} a^{2} T_{1}+\beta_{0}\left(T_{2}\right)$

The solution at order $\varepsilon$ is

$u_{1}=B e^{i \omega_{0} T_{0}}+\frac{(1-9 \alpha)}{8} A^{3} e^{3 i \omega_{0} T_{0}}+c c$

where

$B=\frac{1}{2} b e^{i \gamma}$

This solution is rewritten for real amplitudes and phases

$u_{1}=b \cos \left(\omega_{0} T_{0}+\gamma\right)+\frac{(1-9 \alpha)}{32} a^{3} \cos \left(3 \omega_{0} T_{0}+3 \beta\right)$

Substituting initial conditions for $O(\varepsilon)$

$b(0)=-\frac{(1-9 \alpha)}{32} a_{0}^{3}, \gamma(0)=0$

At $O\left(\varepsilon^{2}\right)$, Equations (38) and (31) are inserted into equation (28) and secular terms are eliminated

$-2 i \omega_{0} D_{1} B-D_{1}^{2} A-2 i \omega_{0} D_{2} A$

$+A \bar{A} B\left(12 \alpha \omega_{0}{ }^{2}-6 \omega_{0}^{2}\right)+\frac{3(1-9 \alpha)}{8} \omega_{0}^{2} \bar{A}^{2} A^{3}(\alpha-1)$

$A^{2} \bar{B}\left(-3 \alpha \omega_{0}^{2}-3 \omega_{0}^{2}\right)-6 \alpha i \omega_{0} A^{2} D_{1} \bar{A}-12 \alpha i \omega_{0} A \bar{A} D_{1} A=0$

If Equations (30), (32), (37) and (39) are used above, one finally has

$a=a_{0}, b=-\frac{(1-9 \alpha)}{32} a_{0}{ }^{3}$,

$\beta=\gamma=-\frac{(3 \alpha-3)}{8} \omega_{0} a^{2} T_{1}-\frac{(3 \alpha-3)}{256} \omega_{0} a_{0}{ }^{4} T_{2}$

The final solution with original variables is

$u=a_{0} \cos \left(\omega_{0} t+\beta\right)$

$+\frac{\varepsilon(1-9 \alpha)}{32} a_{0}^{3}\left[\cos \left(3 \omega_{0} t+3 \beta\right)-\cos \left(\omega_{0} t+\beta\right)\right]+O\left(\varepsilon^{2}\right)$ 


\subsection{Multiple Scales Lindstedt Poincare (MSLP) method}

The time transformation

$$
\tau=\omega t
$$

is applied to the equation (21).

$$
\left(1+3 \varepsilon \alpha u^{2}\right) \omega^{2} u^{\prime \prime}+6 \varepsilon \alpha \omega^{2} u u^{2}+\omega_{0}^{2}\left(u+\varepsilon u^{3}\right)=0
$$

where prime is derivative with respect to new transformed time variable $\tau$. Fast and slow time scales are defined $T_{0}=\tau=\omega t, \quad T_{1}=\varepsilon \tau=\varepsilon \omega t, T_{2}=\varepsilon^{2} \tau=\varepsilon^{2} \omega t(45)$

\section{Using}

$\frac{d}{d \tau}=D_{0}+\varepsilon D_{1}+\varepsilon^{2} D_{2}+\ldots$

$\frac{d^{2}}{d \tau^{2}}=D_{0}^{2}+2 \varepsilon D_{0} D_{1}+\varepsilon^{2}\left(D_{1}^{2}+2 \varepsilon D_{0} D_{2}\right)+\ldots$

and substituting the expansions

$u=u_{0}\left(T_{0}, T_{1}, T_{2}\right)+\varepsilon u_{1}\left(T_{0}, T_{1}, T_{2}\right)+\varepsilon^{2} u_{2}\left(T_{0}, T_{1}, T_{2}\right)+.$.

$\omega_{0}^{2}=\omega^{2}-\varepsilon \omega_{1}-\varepsilon^{2} \omega_{2} \ldots$

into (44) yields after separation

$$
\begin{aligned}
& O(1): \omega^{2} D_{0}{ }^{2} u_{0}+\omega^{2} u_{0}=0 \\
& u_{0}(0)=a_{0}, \quad D_{0} u_{0}(0)=0 \\
& O(\varepsilon): \omega^{2} D_{0}{ }^{2} u_{1}+\omega^{2} u_{1}=-2 \omega^{2} D_{0} D_{1} u_{0}+\omega_{1} u_{0} \\
& -3 \omega^{2} \alpha u_{0}{ }^{2} D_{0}{ }^{2} u_{0}-6 \omega^{2} \alpha u_{0}\left(D_{0} u_{0}\right)^{2}-\omega_{0}{ }^{2} u_{0}{ }^{3} \\
& u_{1}(0)=0,\left(D_{0} u_{1}+D_{1} u_{0}\right)(0)=0 \\
& O\left(\varepsilon^{2}\right): \omega^{2} D_{0}{ }^{2} u_{2}+\omega^{2} u_{2}=-2 \omega^{2} D_{0} D_{1} u_{1} \\
& -\omega^{2}\left(D_{1}{ }^{2}+2 D_{0} D_{2}\right)\left(u_{0}\right)+\omega_{1} u_{1}+\omega_{2} u_{0} \\
& -3 \omega^{2} \alpha\left[u_{0}{ }^{2}\left(D_{0}{ }^{2} u_{1}+2 D_{0} D_{1} u_{0}\right)+2 u_{0} u_{1} D_{0}{ }^{2} u_{0}\right] \\
& -6 \omega^{2} \alpha\left[u_{1}\left(D_{0} u_{0}\right)^{2}+2 u_{0} D_{0} u_{0}\left(D_{0} u_{1}+D_{1} u_{0}\right)\right]-3 \omega_{0}{ }^{2} u_{0}{ }^{2} u_{1}
\end{aligned}
$$

The solution of first order is

$$
u_{0}=A\left(T_{1}, T_{2}\right) e^{i T_{0}}+c c
$$

where

$$
A=\frac{1}{2} a e^{i \beta}
$$

For real amplitude and the phase, the solutions is as follows

$$
u_{0}=a\left(T_{1}, T_{2}\right) \cos \left(T_{0}+\beta\left(T_{1}, T_{2}\right)\right)
$$

Applying the initial conditions

$$
a(0)=a_{0}, \beta(0)=0
$$

Equation (52) is substituted into equation (50) and secular terms are eliminated

$$
-2 i \omega^{2} D_{1} A+A \omega_{1}+3 \omega^{2} \alpha A^{2} \bar{A}-3 \omega_{0}^{2} A^{2} \bar{A}=0
$$

According to the MSLP method, $D_{1} A=0$ is selected firstly and solved. If frequency correction is real number, this choice is acceptable. If $\omega_{1}$ is complex, this choice is not correct. Because, it isn't suitable for physical solutions. Thus, $D_{1} A=0$ is selected and in this case $\omega_{1}$ will be a real number.

$$
D_{1} A=0
$$

which implies $a=a\left(T_{2}\right), \beta=\beta\left(T_{2}\right)$ and $\omega_{1}$ is solved.

$$
\omega_{1}=3 A \bar{A}\left(\omega_{0}^{2}-\alpha \omega^{2}\right)=\frac{3}{4} a^{2}\left(\omega_{0}^{2}-\alpha \omega^{2}\right)
$$

The solution at order $\varepsilon$ is

$$
\begin{aligned}
& u_{1}=B e^{i T_{0}}+\frac{\left(\omega_{0}^{2}-9 \omega^{2} \alpha\right)}{8 \omega^{2}} A^{3} e^{3 i T_{0}}+c c \\
& =b \cos \left(T_{0}+\gamma\right)+\frac{\left(\omega_{0}^{2}-9 \omega^{2} \alpha\right)}{32 \omega^{2}} a^{3} \cos \left(3 T_{0}+3 \beta\right)
\end{aligned}
$$

where

$$
B=\frac{1}{2} b e^{i \gamma}
$$

The initial conditions is applied for Equation(59), one has

$$
b(0)=-\frac{\left(\omega_{0}^{2}-9 \omega^{2} \alpha\right)}{32 \omega^{2}} a_{0}^{3}, \gamma(0)=0
$$

At the last order, substitution of (52) and (59) into the right hand side of (51) and secularities are eliminated

$$
\begin{aligned}
& -2 i \omega^{2} D_{2} A+A \omega_{2}+\left(-3 \omega_{0}^{2}+3 \omega^{2} \alpha\right) A \bar{A} B \\
& +\left(-3 \omega_{0}^{2}+3 \omega^{2} \alpha\right) A^{2} \bar{B} \\
& +\left(\omega_{0}^{2}-9 \omega^{2} \alpha\right)\left(\frac{-3 \omega_{0}^{2}+3 \omega^{2} \alpha}{8 \omega^{2}}\right) A^{3} \bar{A}^{2}=0
\end{aligned}
$$

According to the MSLP, $D_{2} A=0$ is selected firstly. This choice is admissible because $\omega_{2}$ is real for this choice. After algebraic manipulations, equation (62) yields

$$
\begin{aligned}
& a=a_{0}, b=-\frac{\left(\omega_{0}{ }^{2}-9 \omega^{2} \alpha\right)}{32 \omega^{2}} a_{0}{ }^{3}, \quad \beta=\gamma=0, \\
& \omega_{2}=\frac{30 \alpha \omega^{2} \omega_{0}{ }^{2}-27 \alpha^{2} \omega^{4}-3 \omega_{0}{ }^{4}}{128 \omega^{2}} a_{0}{ }^{4}
\end{aligned}
$$

The frequency is

$$
\begin{aligned}
& \omega^{2}=\omega_{0}^{2}+\varepsilon \frac{3}{4} a_{0}^{2}\left(\omega_{0}^{2}-\alpha \omega^{2}\right)+ \\
& \varepsilon^{2}\left(\frac{30 \alpha \omega^{2} \omega_{0}^{2}-27 \alpha^{2} \omega^{4}-3 \omega_{0}^{4}}{128 \omega^{2}}\right) a_{0}{ }^{4}
\end{aligned}
$$

Frequency is solved 
$\omega=\sqrt{-\frac{1}{2} z+\frac{1}{2} \sqrt{z^{2}-12 \varepsilon^{2} \frac{\omega_{0}{ }^{2} a_{0}{ }^{4}}{128+96 \varepsilon a_{0}{ }^{2} \alpha+27 \varepsilon^{2} \alpha^{2} a_{0}{ }^{4}}}}$

where

$$
z=\frac{-128-96 \varepsilon a_{0}^{2}-30 \varepsilon^{2} \alpha a_{0}{ }^{4}}{128+96 \varepsilon a_{0}^{2} \alpha+27 \varepsilon^{2} \alpha^{2} a_{0}^{4}}
$$

The final solution is

$$
\begin{aligned}
& u=a_{0} \cos (\omega t)+ \\
& \varepsilon \frac{\left(\omega_{0}^{2}-9 \alpha \omega^{2}\right)}{32 \omega^{2}} a_{0}^{3}(\cos (3 \omega t)-\cos (\omega t))+O\left(\varepsilon^{2}\right)
\end{aligned}
$$

\section{Results and Discussion}

In this section, approximate analytical solutions of classical MS method and MSLP method are contrasted with numerical integration solution obtained by integrating directly the nonlinear ordinary differential equation numerically using the Runge-Kutta method, a built-in function in MATHEMATICA.

To verify the results, time histories of the MS and MSLP methods are compared with the numerical simulations.

In all comparisons, $a_{0}=1, m=1 \quad$ and $\xi=0.1\left(k_{1}=50, k_{3}=5\right)$ are selected. In Figure 2, approximate solutions are compared with numerical simulations for weakly nonlinear systems. For this choice $\lambda=0.5$ is selected first. The Figure illustrates that approximate solutions are in excellent agreement with numerical simulations. In Figures 3 and 4, the effect of cubic nonlinearity is amplified by increasing $\lambda$. In Figure 3, separations are observed. MSLP is better aligned with numerical solutions for $\lambda=5$. Finally, in Figure $4, \lambda=25$ is selected. For this strong nonlinear case, While MSLP and numerical solutions have a good agreement, approximate frequency of MS solution is very different from the numerical solution.

To further show the accuracy of present (MSLP) method, a comparison of angular frequencies obtained by different methods is presented in Table 1 for weak and strong nonlinearities. In Table 1, approximate frequency of the present (MSLP) method and the existing results are in excellent agreement with numerical results for weak nonlinearities. Apart from the approximate results obtained by Telli and Kopmaz [14], approximate frequency of MSLP method and existing results have excellent agreement with numerical for strong nonlinear systems.

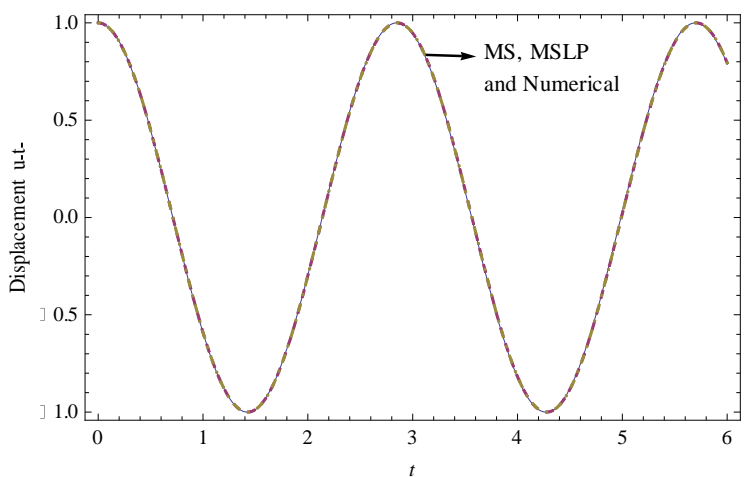

Figure 2. Comparison of approximate solutions and numerical solution for $\lambda=0.5, \xi=0.1\left(k_{1}=50, k_{3}=5\right)$

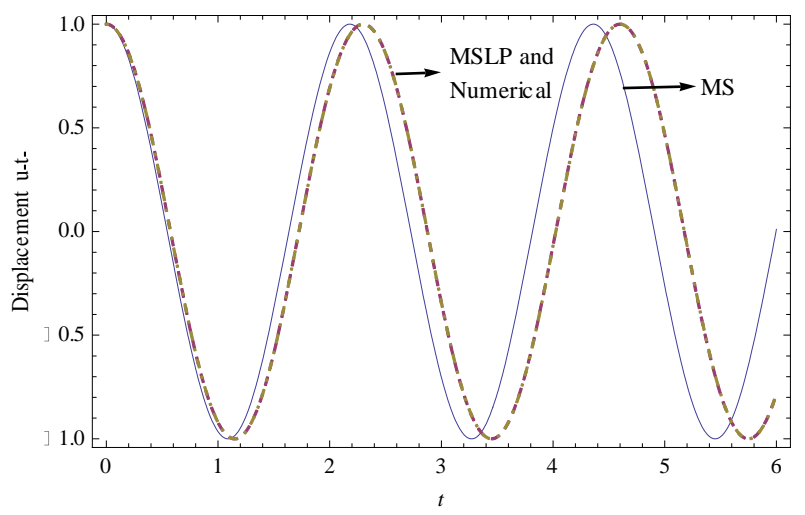

Figure 3. Comparison of approximate solutions and numerical solution for $\lambda=5, \xi=0.1\left(k_{1}=50, k_{3}=5\right)$

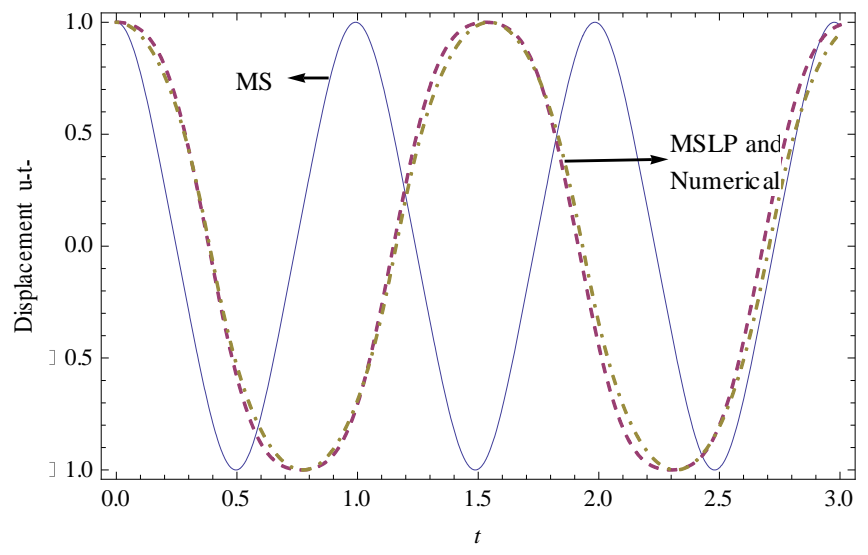

Figure 4. Comparison of approximate solutions and numerical solution for $\lambda=25, \xi=0.1\left(k_{1}=50, k_{3}=5\right)$ 


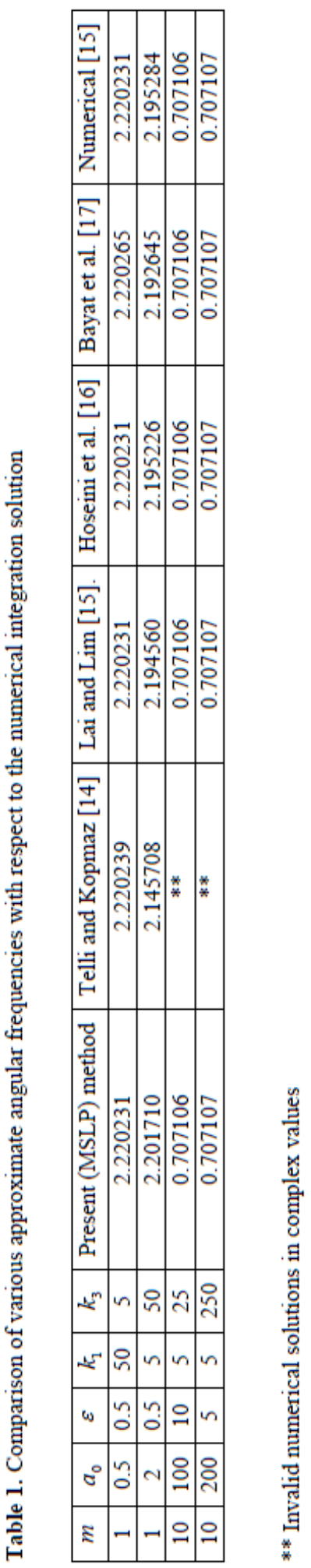

\section{Conclusions}

MS and MSLP method from the combination of MS and Lindstedt Poincare methods are applied to system with free vibration of a mass with serial linear and nonlinear stiffness on a frictionless surface. The time histories of MS and MSLP methods are compared with numerical solutions. Although the solution of MSLP and numerical are in excellent agreement, MS solution is not valid for strong nonlinear systems. Approximate frequency of MSLP method and previous results are contrasted with numerical solutions. The comparison shows that the results of MSLP is valid on a wide range of system parameters considered. In addition, the MSLP is appropriate not only for conservative systems but also for non-conservative systems.

\section{References}

1. Nayfeh, A.H, Mook, D.T, Nonlinear Oscillations; John Wiley and Sons: New York, 1979; pp 720.

2. Nayfeh, A.H, Introduction to Perturbation Techniques; John Wiley and Sons: New York, 1981; pp 532.

3. Mickens, R.E, Oscillations in Planar Dynamic Systems; Word Scientific: New York, 1996; pp 340.

4. He, J.H, Linearized perturbation technique and its applications to strongly nonlinear oscillators, Computers and Mathematics with Applications, 2003, 45, 1-8.

5. $\mathrm{Hu}, \mathrm{H}, \mathrm{A}$ classical perturbation technique which is valid forlarge parameters, Journal of Sound and Vibration, 2004, 269, 409-412.

6. $\mathrm{Xu}, \mathrm{L}$, Determination of limit cycle by He's parameter-expanding method for strongly nonlinear oscillators, Journal of Sound and Vibration, 2007, 302, 178-184.

7. He, J.H, Homotopy perturbation method: a new nonlinear analytical technique, Applied Mathematics and Computation. 2003, 135, 73-79.

8. Pakdemirli, M, Karahan, M.M.F, Boyac1, H, A new perturbation algorithm with better convergence properties: multiple scales Lindstedt Poincare method, Mathematical and Computational Applications, 2009, 14, 31-44.

9. Pakdemirli, M, Karahan, M.M.F, A new perturbation solution for systems with strong quadratic and cubic nonlinearities, Mathematical Methods in the Applied Sciences, 2010, 33, 704-712.

10. Pakdemirli, M, Karahan, M.M.F, Boyac1, H, Forced vibrations of strongly nonlinear systems with multiple scales Lindstedt Poincare method, Mathematical and Computational Applications, 2011, 16, 879-889.

11. Karahan, M.M.F, Pakdemirli, M, Free and forced vibrations of the strongly nonlinear cubic-quintic Duffing oscillators, Zeitschrift für Naturforschung A, 2017, 72(1), 59-69.

12. Karahan, M.M.F, Pakdemirli, M, Vibration analysis of a beam on a nonlinear elastic foundation, Structural Engineering and Mechanics, 2017, 62(2), 171-178.

13. Karahan, M. M. F, Approximate solutions for the nonlinear third-order ordinary differential equations, Zeitschrift für Naturforschung $A$, 2017, 72(6), 547-557. 
14. Telli, S, Kopmaz, O, Free vibrations of a mass grounded by linear and nonlinear springs in series, Journal of Sound and Vibration, 2006, 289, 689-710.

15. Lai, S.K, Lim, C.W, Accurate approximate analytical solutions for nonlinear free vibration of systems with serial linear and nonlinear stiffness, Journal of Sound and Vibration, 2007, 307, 720-736.

16. Hoseinia, S.H, Pirbodaghi, T, Asghari, M, Farrahi, G.H, Ahmadian, M.T, Nonlinear free vibration of conservative oscillators with inertia and static type cubic nonlinearities using homotopy analysis method, Journal of Sound and Vibration, 2008, 316, 263-273.
17. Bayat, M, Bayat, M, Bayat, M, An analytical approach on a mass grounded by linear and nonlinear springs in series, International Journal of the Physical Sciences, 2011, 6(2), 229-236.

18. Inman, D.J, Engineering Vibration; Prentice Hall: New Jersey, 1996; pp 667. 